\title{
Influence of lane departure warnings onset and reliability on car drivers' behaviors
}

Running title: Lane departure warning onset and reliability

J. Navarro' ${ }^{1}$ J. Deniel ${ }^{1}$, E. Yousfi ${ }^{1}$, C. Jallais ${ }^{2}$, M. Bueno² \& A. Fort ${ }^{2}$

${ }^{1}$ Laboratoire d’Etude des Mécanismes Cognitifs (EA 3082), University Lyon 2, France

2 LESCOT-TS2-IFSTTAR (French Institute of Science and Technology for Transport, Development and Networks), Bron, France

Address correspondence to Jordan Navarro, Institut de Psychologie, 5 avenue Pierre Mendès-France, 69676 Bron Cedex, France, jordan.navarro@univ-lyon2.fr

Pour citation :

NAVARRO, Jordan, DENIEL, Jonathan, YOUSFI, Elsa, JALLAIS, Christophe, BUENO, Mercedes, FORT, Alexandra, 2017, Influence of lane departure warnings onset and reliability on car drivers' behaviors, Applied Ergonomics, 59, Part A, Elsevier, pp. 123-131, DOI: 10.1016/ j.apergo.2016.08.010 


\section{ABSTRACT}

Lane departures represent an important cause of road crashes. The objective of the present study was to assess the effects of an auditory Lane Departure Warning System (LDWS) for partial and full lane departures (onset manipulation) combined with missed warnings (reliability manipulation: $100 \%$ reliable, $83 \%$ reliable and $66 \%$ reliable) on drivers' performances and acceptance. Several studies indicate that LDWS improves drivers' performances during lane departure episodes. However, little is known about the effects of the warning onset and reliability of LDWS. Results of studies which looked at forward collision warning systems show that early warnings tend to improve drivers' performances and receive a better trust judgement from the drivers when compared to later warnings. These studies also suggest that reliable assistances are more effective and trusted than unreliable ones. In the present study, lane departures were brought about by means of a distraction task whilst drivers simulated driving in a fixed-base simulator with or without an auditory LDWS. Results revealed steering behaviors improvements with LDWS. More effective recovery maneuvers were found with partial lane departure warnings than with full lane departure warnings and assistance unreliability did not impair significantly drivers' behaviors. Regarding missed lane departure episodes, drivers were found to react later and spend more time out of the driving lane when compared to properly warned lane departures, as if driving without assistance. Subjectively, LDWS did not reduce mental workload and partial lane departure warnings were judged more trustworthy than full lane departure ones. Data suggests the use of partial lane departure warnings when designing LDWS and that even unreliable LDWS may draw benefits compared to no assistance.

Keywords: steering, lane departure warning, alert timing, alert miss, driving simulator 
Among the several attempts made in the past few decades to decrease car crashes and related injuries, is the attempt to assist people whilst driving. A variety of driving assistance devices have since been designed and implemented in cars. Different devices were developed to help drivers carry out the three driving sub-tasks: (i) navigation - e.g. navigation aids using global positioning system; (ii) hazard identification - e.g. collision warning; and (iii) control e.g. adaptive cruise control for longitudinal control or electronic stability program for lateral control - (Stanton et al., 2001).

According to road crashes analyses, lane departures are one of the leading causes of crashes, representing between 14.5\% (ADAC, 2001; Pohl et al., 2007) and 40\% (Bar and Page, 2002) of the causes of all crashes that occurred in different European countries. The contribution of lane departure episodes to fatal crashes is even more significant, representing between 35\% (ADAC, 2001; Pohl et al., 2007) and 70\% (Bar and Page, 2002) of the causes of all fatal crashes. In the United States, lane departures were also reported to be a factor in $66 \%$ of crashes involving a single light vehicle (Najm et al., 2007) and caused 28\% of fatal crashes in 2005 (National Highway Traffic Safety Administration, 2006). Considering all light vehicle crashes together, more than $27 \%$ of crashes are due to lane departures, corresponding to more than $30 \%$ of crashes economic cost, and 36\% of functional years lost (Najm et al., 2007).

Assistances considered here are meant to assist drivers in the lateral control sub-task. A classification based on a mixture of two theoretical frameworks (Hoc et al., 2009; Young et al., 2007) and a review of all lateral control assistance devices is available (Navarro et al., 2011). According to this classification, Lane Departure Warning Systems (LDWS) belong to a mutual control mode where both the driver and the assistance share the supervision of lateral control but only the driver remains in charge of steering. The assistance only warns the driver when a given lateral position is judged as unsafe. 
LDWS can be considered as an extension of the rumble strip concept. If rumble strips are known to be effective to reduce crashes (e.g. Auberlet et al., 2010; Hatfield et al., 2009; Meuleners et al., 2011; Morena, 2003), they did not offer much onset flexibility and variety of design options offered by LDWS, such as the sensorial modality used to convey the warning signal and its duration (Navarro et al., 2011).

LDWS are considered to have the potential for preventing crashes and particularly serious crashes. More precisely, LDWS are described as relevant to prevent or mitigate between 146000 and 179000 crashes per year in the United States including between 4842 and 7529 fatal crashes (Jermakian, 2011). Several studies indicate that LDWS improve drivers' performances when facing lane departure episodes. Indeed compared to no assistance, LDWS were found to reduce steering reaction times (Deroo et al., 2013, 2012; Kozak et al., 2006; Navarro et al., 2007; Rossmeier et al., 2005), and the number, magnitude and duration of lateral excursions (Deroo et al., 2013, 2012; Hoc et al., 2006; Navarro et al., 2010, 2007; Rimini-Döring et al., 2005). These studies indicate that LDWS are improving safety during lane departure episodes. These findings are confirmed by a naturalistic study where two distinct cohort of trucks equipped with LDWS were compared with trucks not equipped with LDWS, over 13 billion miles traveled. Trucks without LDWS had a crash rate approximately 2 times higher compared to those with LDWS (Hickman et al., 2015).

LDWS are assumed to improve drivers' situation diagnosis through their ability to capture a driver's attention (Ho et al., 2005; Spence and Ho, 2008). However, situation diagnosis also implies that drivers must make a cognitive assessment of the situation, taking into account various contextual elements, before acting (Kovordányi et al., 2005; Navarro et al., 2007). Thus, situation diagnosis requires some time to be completed and is dependent on both the context of the situation and the design of the warning signal delivered. As a consequence, and in order to optimize the benefits of the LDWS, the onset of the warning delivery is of particular interest. Situation diagnosis might also be under the influence of the LDWS reliability as assistance reliability is known to affect human dependence on assistance (Lee and Moray, 1992; Muir, 
1994; Parasuraman and Riley, 1997). Individuals are more likely to depend on perfectly or highly reliable assistances than on poorly reliable assistances. Following this rationale, assistances with less than approximately $70 \%$ of reliability were found to be worse than no assistance (Wickens and Dixon, 2007). Even artificially low or high statements about assistance reliability were reported to have long-lasting influence on the perception of assistance performance (Barg-Walkow and Rogers, 2016). However, surprisingly little is known about the effects of lane departure warning onset and reliability on driving performances, and its acceptance. The aim of the experiment was to address these two points.

In a test-track experiment manipulating LDWS onset, it was found that an early onset resulted in more warnings, fewer lane departures and a lower acceptance than a late onset (Tijerina et al., 1996). These results were confirmed recently; full lane departure warnings were found less effective but more accepted than partial lane departures (Navarro et al., 2016). These findings are consistent with those gained from a series of driving simulator studies looking at Forward Collision Warning Systems (FCWS, longitudinal control assistance while following a car). Indeed, early warnings tend to decrease braking reaction times compared to later warnings particularly for urgent braking (Abe and Richardson, 2006a, 2006b, 2005, 2004). However, contrary to LDWS, early warnings received a better trust judgement from drivers. This data reinforces previous work that showed the efficiency of early warnings to help distracted drivers to react more quickly than with late FCWS or no assistance (Lee et al., 2002). A too quickly drawn conclusion would be that the earlier the warning the better, as early warnings should give drivers more time to react to hazardous situations in addition to helping with the situation diagnosis. However, it is known that the earlier the warning the more likely it is that the warning will be considered as a false alert (McGehee et al., 2002; Parasuraman et al., 1997; Shinar, 1978). As a result, warning signals considered as false alarms might be judged as harmful and potentially ignored by drivers (Abe and Richardson, 2006a, 2004). Thus warning onset and reliability can hardly be considered independently in practice. This is even more true for LDWS compared to FCWS. If a FCW is judged as too early it may be considered by drivers as 
a very careful warning delivery, whereas a too early LDW may be perceived as a false warning because the current lateral position of the vehicle is perceived as completely safe. This would explain why early warnings are judged as acceptable with FCWS (Abe and Richardson, 2006a, 2005, 2004) and unacceptable with LDWS (Navarro et al., 2016; Tijerina et al., 1996).

Driving assistance designers tend to minimize the risk of missed warnings (Parasuraman et al., 2000). Missed warnings are considered as more harmful than false warnings (i.e. false warnings just have to be ignored whereas missed warnings may put the operator at risk immediately). Minimizing the risk of false warnings necessarily implies an increase of false warnings (Parasuraman et al., 2000). Therefore, initially our focus was on LDWS false warnings (Navarro et al., 2016). It was found that the unreliable LDW (33\% and 17\% of invalid warnings) delivering false warnings alone or combined with missed warnings did not affect significantly driving performances during lane departure episodes compared to an unassisted control condition.

In practice however, drivers are not necessarily aware of the LDWS presence and operational status in their vehicle (Braitman et al., 2010; Robinson et al., 2011). Those results are complemented by a survey conducted on 343 UK, Swedish and Chinese drivers that were asked to assess the usefulness of 10 "information enhancement systems" on a five-point scale from 1 (not useful) to 5 (very useful). LDWS were evaluated as the less useful assistance with 2,67 points on the average (Duan and Chen, 2011). Additionally, among various assistance devices (i.e. FCWS with autobrake, LDWS, side-view assist, and/or active bi-xenon headlights), LDWS were judged the most annoying assistance (Braitman et al., 2010). Only 59\% of driver's declared to keep the LDWS turned on whilst driving (Eichelberger and McCartt, 2014). Due to LDWS being an assistance drivers are able to control (i.e., turn on/off), the actual effectiveness of LDWS is dependent on driver acceptance and related use of it. In everyday life, drivers tend to disengage LDWS because some of the delivered warnings are perceived as false warnings by drivers who ultimately found LDWS annoying. If disengaged, LDWS are obviously useless. A solution would be to reduce as much as possible the risk of false warnings in order to deliver 
only valid warnings. This solution would most likely result in LDWS no longer being judged as annoying by drivers, however, the risk of missed warnings would necessarily increase.

Furthermore, LDWS are based on lane marking detection to trigger warnings and cannot be used in the absence of road markings. Even with road markings, not all lane departures can be detected due to snow, heavy rain, fog, ice or technical problems, for example. As a matter of fact, the question that remains to be addressed is the impact of missed warnings on drivers' performances during the situation of the missed lane departure detection and the following lane departures. Based on Muir's automation trust model, an unreliable assistance should lead drivers to reject the assistance considered (Muir, 1994). Such adaptive behaviors were not observed in both simulated and real world environments, where reliable and unreliable (false positives and negatives) LDWS were showed to improve lane keeping performances by preventing drivers to approach lane-edge zones (Rudin-Brown and Noy, 2002). Drivers' trust regarding the LDWS was greater with the perfectly reliable device than with an unreliable one. However, no data was reported regarding the lane departure episodes. Results gained from FCWS indicate that unreliable warnings can dramatically reduce the benefits of the device (Bliss and Acton, 2003; Enriquez and MacLean, 2004). In a car following task, drivers' reaction times to the lead car braking increased with an unreliable collision warning system (false and missed warnings) compared to a reliable one and were even longer than without assistance. Furthermore, longer reaction times for the lead vehicle brake situations following assistance errors were recorded (Abe et al., 2002). Notwithstanding this, drivers also spent less time in the hazardous zone, too close from the followed vehicle, whatever the reliability of the FCWS.

It seems that reliable assistances are more effective than unreliable ones. However, there is a risk that drivers could rely too much on reliable assistance, leading to a drop in driving performances. This drop was observed with FCWS in terms of longer reaction times (Abe et al., 2002) and quality of the reaction (Maltz and Shinar, 2007) to a hazardous event, but it could also result in more crashes. Indeed, more crashes were observed with a perfectly reliable assistance than with an imperfectly reliable assistance (Bliss and Acton, 2003). As a 
consequence, unreliable FCWS were not always found less effective than reliable ones. Additionally, a large number of missed warnings (Maltz and Shinar, 2004) and various warning reliability levels (60\%, 70\%, 80\%, 95\% and 100\%) (Ben-yaacov et al., 2002; Bueno et al., 2012) did not have any significant impact on drivers' behaviors.

The objective of the present study was to assess the effects of an auditory LDWS for partial and full lane departure (onset manipulation) combined with missed warnings (reliability manipulation: $100 \%$ reliable, $83 \%$ reliable and $66 \%$ reliable) on drivers' performances and acceptance. Partial lane departure and/or reliable warnings were expected to be more effective and more accepted by drivers than full lane departure and/or unreliable warnings. Missed warning lane departures were expected to weaken drivers' performances, compared to no assistance, and the benefits of assistance for the subsequent lane departures were expected to lower.

\section{METHOD}

\subsection{Participants}

Three equivalent groups of twelve participants (18 females and 18 males), aged from 24 to 44 years (mean age $=30.7$ years \pm 5.2 ), with driving experience ranging from 3 to 26 years (mean $=12.1$ years \pm 5.7 ), took part in the experiment. All of the participants had normal or corrected-to-normal vision. No participants experienced motion sickness. Each received a financial compensation of 50 euros for their participation.

\subsection{Simulator}

The experiment took place on a fixed-base simulator (developed by LEPSIS-COSYSIFSTTAR). The visual environment was projected onto seven screens providing a horizontal angle of about $300^{\circ}$ around the driver. The simulator cabin was a Peugeot 308 equipped with a 
manual gearbox, a force feedback steering wheel, pedals for brakes, accelerator and clutch, a speedometer and cruise control set at $90 \mathrm{kph}$. The visual database consisted of a model of a straight three-lane road developed for the purpose of the experiment.

\subsection{Distraction task}

Lane departures were brought about by means of a reading task - words were displayed on a 7 -inche screen placed on the dashboard $\left(25^{\circ}\right.$ down and $35^{\circ}$ right from the driver gaze straight ahead).

The screen displayed four words at a time, which refreshed every second, from a total of 3097 words selected from the MANULEX database (Lété et al., 2004) based on their length (between 6 and 9 letters) and use frequency (50\% of the words closer to the frequency average). Between 1 and 4 infinitive verbs were presented with an average of 2 infinite verbs every eight words displayed.

Whilst driving, participants were instructed to read aloud as many verbs as possible without looking at the road for the period of the distraction task. A camera recording drivers' faces and eyes was used to check that drivers kept their eyes on the distraction task.

During each driving scenario, twelve distraction tasks were played, separated by 20 to 40 seconds between each one. Half of the distraction tasks led to a lane departure (3 on the right and 3 on the left). To ensure that the distraction tasks lead to a left or a right lane departure or to no lane departure, the driving simulation was manipulated during the reading task. For those distraction tasks that should not lead to a lane departure, the vehicle trajectory was maintained in the driving lane. For those distraction tasks that should lead to a lane departure, the vehicle trajectory was slightly changed $\left(1^{\circ}\right.$ of vehicle heading) to the right or to the left to ensure a left or a right lane departure respectively. The trajectory change had no effect on the steering wheel force feedback. Because the vehicle heading was changed during the distraction task, it was not possible for the participants to notice those manipulations and post experiment interviews confirmed this. 
During the manipulation of the driving simulation, drivers kept control of the steering wheel and were able to steer the car normally. Because drivers were asked to focus on a very demanding distraction task and were instructed to keep both hands on the steering wheel during those episodes and drive in a straight line, the lane departures appeared as natural. Pretests were used to set the manipulations of the driving simulation to ensure that provoked and prevented lane departures were not perceived as artificial and unexpected.

The distraction task stopped when a Partial Lane Departure -PLD- or Full Lane Departure FLD- was reached depending on the condition (onset manipulation). PLD was reached as soon as part of the front tyre touched the inside edge of the closest lane marking. FLD was reached when the front tyre was completely beyond the outside edge of the lane marking.

When the distraction task ended, the 7-inch screen used to display the words remained black. In the case of a driving scenario without assistance, no further information was provided. In the case of a driving scenario with driving assistance, the end of the distraction task was associated with a warning.

\subsection{Driving assistance device}

The driving assistance device consisted of a sound similar to a rumble strip noise played from the loudspeakers of the simulator cabin when the vehicle was leaving its lane. The sound remained active as long as the car was not driven back into its lane.

The end of the distraction task (either PLD or FLD) and the warning were triggered at the same time. A control condition without assistance was performed for both PLD and FLD. For those conditions, no warning was delivered at the end of the distraction task.

Each group of twelve participants was given a reliability level (Perfect -100\%-, Medium $83 \%$ - or Low -66\%-) to ensure that the percentage of assistance reliability was maintained for each participant for the entire duration of the experiment (i.e. partial and full lane departure onsets). 
All in all, each participant took part in four successive driving scenarios balanced in each group of participants using Latin square design: (1) No Assistance with Partial Lane Departure; (2) No Assistance with Full Lane Departure; (3) Assistance with Partial Lane Departure; and (4) Assistance with Full Lane Departure.

\subsection{Procedure}

Drivers were instructed to drive in the middle lane of the three-lane road, to keep both hands on the steering wheel and to reach the speed of $90 \mathrm{kph}$. When the speed of $90 \mathrm{kph}$ was reached, cruise control was automatically engaged and the speed of $90 \mathrm{kph}$ was maintained for the remainder of the driving scenario. Cruise control was used in order to ensure similar lane departure episodes.

The experiment lasted approximately an hour, beginning with a training scenario which aimed to familiarize drivers with the simulator, the distraction task and the procedure. The experiment itself consisted of 4 driving scenarios (approximately 8 minutes/scenario), each followed by questionnaires. Drivers had to complete the NASA-TLX in order to assess subjective workload (Hart and Staveland, 1988) and a questionnaire of acceptance of the assistance device (Reagan and Bliss, 2013) when the assistance was present.

Prior to the experiment participants were presented with the assistance device and the warning sound. The assistance device was not experienced in driving conditions before the experiment. Participants were not informed of the assistance device reliability and onset manipulations.

The two groups of participants with an imperfect driving assistance faced one miss $(83 \%$ reliable) or two misses (66\% reliable) among the six lane departures induced through the distraction task. The misses occurred after the second or third distraction task (and first lane departure) for the medium reliability (83\%) and after the second or third and the fifth or sixth distraction task (and first and third lane departure) for the low reliability (66\%). 
Traffic on both ways was present at a rate of approximately six vehicles per kilometer. However, the traffic was arranged in such a way that drivers never had to take into account a potential risk of collision nor to change lane.

\subsection{Data analysis}

Newman-Keuls tests were used for post-hoc comparisons. The level of significance of $p<0.05$ was used in all tests.

\subsubsection{Driving behaviors during lane departure episodes}

In order to assess driving behaviors during lane departure episodes, the Duration of Lateral Excursion (DLE) was computed. DLE is the time spent by drivers outside of their driving lane (i.e. the most outward portion of the vehicle over the centerline of the lane boundary) during a provoked lane departure. Steering reaction times that corresponded to the time elapsed between the end of the distraction task and the moment when drivers began to turn the steering wheel was also calculated.

\subsubsection{Lane departures without the warning missed situations}

A 3 × 2 × 2 mixed design ANOVA with one between-participants factor (reliability level: 100\%, 83\%, 66\%) and two within-participants factors (onset: Partial Lane Departure, Full Lane Departure; driving assistance: no assistance, assistance) was used to assess lane departures without the warning missed situations.

\subsubsection{Missed warning lane departures}

Analysis was carried out in order to assess the lane departures missed by the assistance device compared to no assistance and lane departures with a perfectly reliable assistance. A $3 \mathrm{x}$ 2 mixed design ANOVA with one between-participants factor (influence of miss: behavior when the assistance is perfectly reliable, during the miss with assistance reliability of $83 \%$, during the first miss with assistance reliability of $66 \%$, during the second with assistance reliability of $66 \%$ 
and with no assistance) and one within-participants factor (onset: Partial Lane Departure, Full Lane Departure) was adopted.

\subsubsection{The lane departure following a missed warning}

An analysis was then performed to assess behaviors for the lane departure following a lane departure missed as compared to no assistance and lane departures with a perfectly reliable assistance. A $3 \times 2$ mixed design ANOVA with one between-participants factor (influence of miss: behavior when the assistance is perfectly reliable, after the miss with assistance reliability of $83 \%$, after the first miss with assistance reliability of $66 \%$, after the second miss with assistance reliability of $66 \%$ and with no assistance) and one within-participants factor (onset: Partial Lane Departure, Full Lane Departure) was performed.

\subsubsection{Global lateral position}

A $3 \times 2 \times 2$ mixed design ANOVA with one between-participants factor (reliability level: $100 \%, 83 \%, 66 \%$ ) and two within-participants factors (onset: Partial Lane Departure, Full Lane Departure; driving assistance: no assistance, assistance) was used to assess driving behaviors outside of the distraction tasks and associated lane departures on a dependent variable assessing drivers' position in the driving lane. To do so, a variable inspired from Land (the surface between the lane center and the car center) was computed for all road sections with no distraction tasks or lane departure (Land and Horwood, 1995).

\subsubsection{Subjective mental workload and assistance acceptance}

A 3 × 2 × 2 mixed design ANOVA with one between-participants factor (reliability level: $100 \%, 83 \%, 66 \%$ ) and two within-participants factors (onset: Partial Lane Departure, Full Lane Departure; driving assistance: no assistance, assistance) was used to assess subjective workload.

Finally, a 3 x 2 mixed design ANOVA with one between-participants factor (reliability level: 100\%, 83\%, 66\%) and one within-participants factors (onset: Partial Lane Departure, Full Lane Departure) was used to assess assistance acceptance. 


\subsection{Lane departures without the warning missed situations}

\subsubsection{Duration of lateral excursion (DLE)}

The assistance device reliability did not significantly influence the mean $\operatorname{DLE}(F(2,33)=0.29$, $p=.75$ ). Mean DLE were significantly reduced in the presence of the assistance device compared to the no assistance condition (assistance: $4.45 \mathrm{~s}$, no assistance: $5.64 \mathrm{~s}, F(1,33)=92.62, p<.001$, see Fig. $1 \mathrm{~A})$. The onset also impacted significantly the $\operatorname{DLE}(F(1,33)=5.38, p<.03)$ and a significant interaction between onset and the presence of assistance was found $(F(1,33)=4.87$, $p<.04)$. Post-hoc analyses revealed that the assistance device is significantly more valuable for PLD (4.17s) than for FLD (4.73s) whereas PLD and FLD did not significantly impact DLE without assistance (PLD: 5.63s, FLD: 5.64s).

\subsubsection{Steering reaction time}

The assistance device reliability did not significantly influence the mean steering Reaction Times (RTs) $(F(2,33)=0.51, p=.61)$. Mean RTs were significantly reduced in the presence of the assistance device compared to the no assistance condition (assistance: $0.88 \mathrm{~s}$, no assistance: 1.18 s, $F(1,33)=30.99, p<.001$, see Fig. 1B). The onset of the warning also impacted significantly 
RTs (PLD: 0.92s, FLD: 1.14s, $F(1,33)=26.14, p<.001)$.

\section{$-A-$}

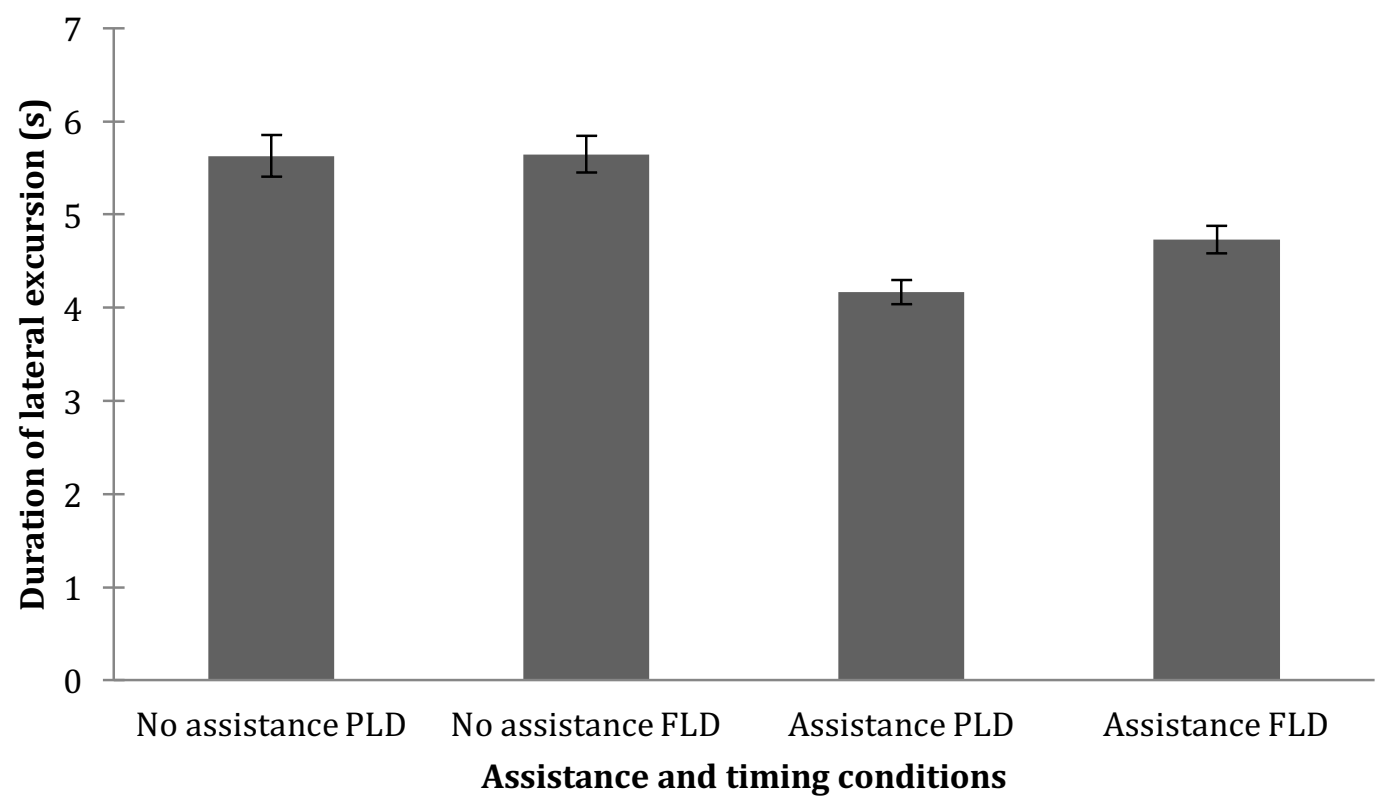

-B-

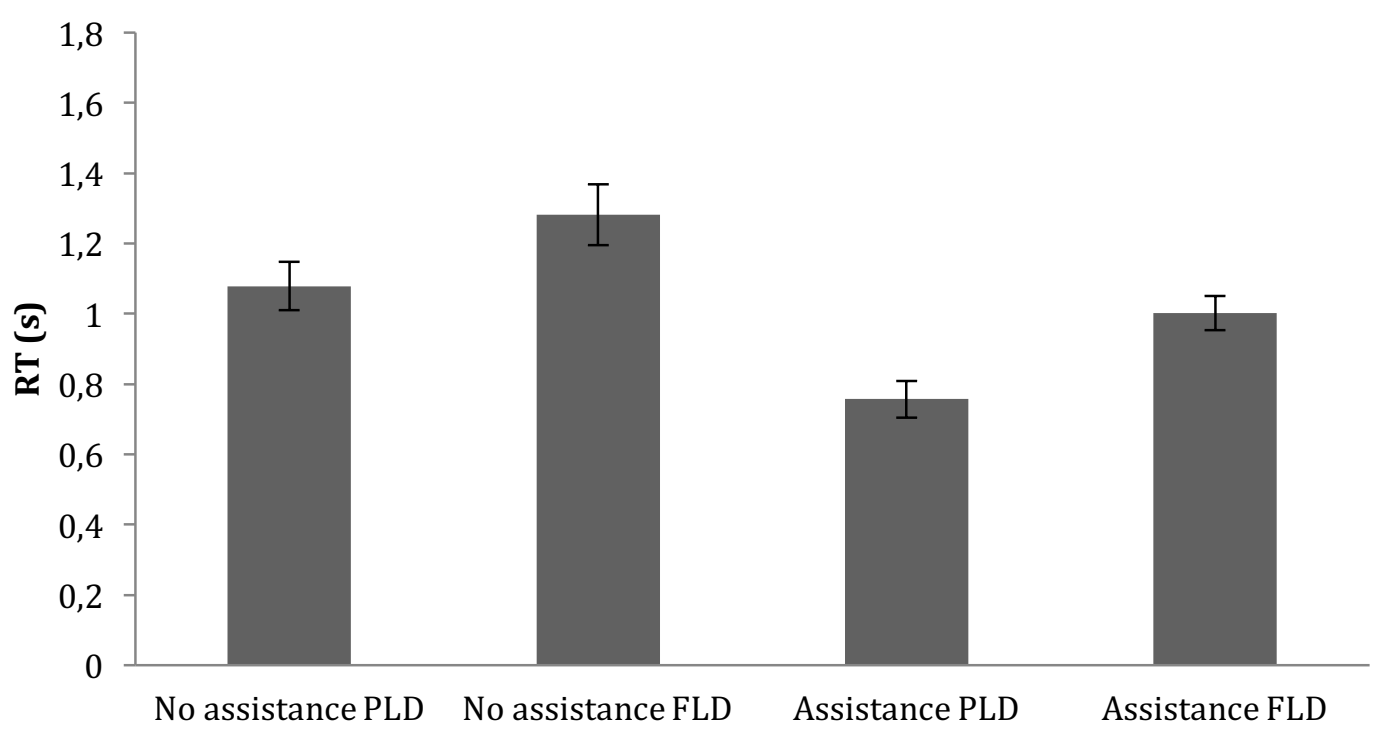

Assistance and timing conditions

Figure 1: Mean duration of lateral excursion $(A)$ and mean steering reaction time (B) for partial (PLD) and full (FLD) lane departure episodes, missed situations excluded. Error bars represent standard errors. 


\subsection{Missed warning lane departures}

\subsubsection{Duration of lateral excursion}

Figure 2A shows the mean DLE for the warnings missed (miss 83\% reliability assistance, miss $166 \%$ reliability assistance and miss $266 \%$ reliability assistance) comparatively with the average DLE with the perfectly reliable assistance and the no assistance condition. An overall significant effect was observed $(F(4,52)=6.78, p<.001)$. Post-hoc analyses indicated a significant DLE difference between lane departures properly signaled with a perfectly reliable assistance and the first warning missed either with the 83\% and the 66\% (Miss $166 \%$ ) assistances. However, this difference was not significant for the second warning missed (Miss $266 \%$ ). In the case of this second warning missed, DLE are significantly reduced compared to the DLE observed during the first warning missed (miss $83 \%$ and miss $166 \%$ ). More precisely, DLE observed for that second miss (4.92s) were positioned half way between the DLE observed with the perfectly reliable assistance (4.28s) and the DLE observed in the no assistance condition (5.64s) without significantly differing from one or the other.

\subsubsection{Steering reaction time}

Similarly to what was observed for DLE, an overall significant effect was observed for RTs $(F(4,52)=3.62, p<.02$, Fig. 2B). However, post-hoc analyses only indicated a statistical RT difference between lane departures properly signaled with a perfectly reliable assistance and the first warning missed in the 83\% $(p<.05)$ and a trend in the $66 \%$ (Miss $166 \%, p<.1$ ). 


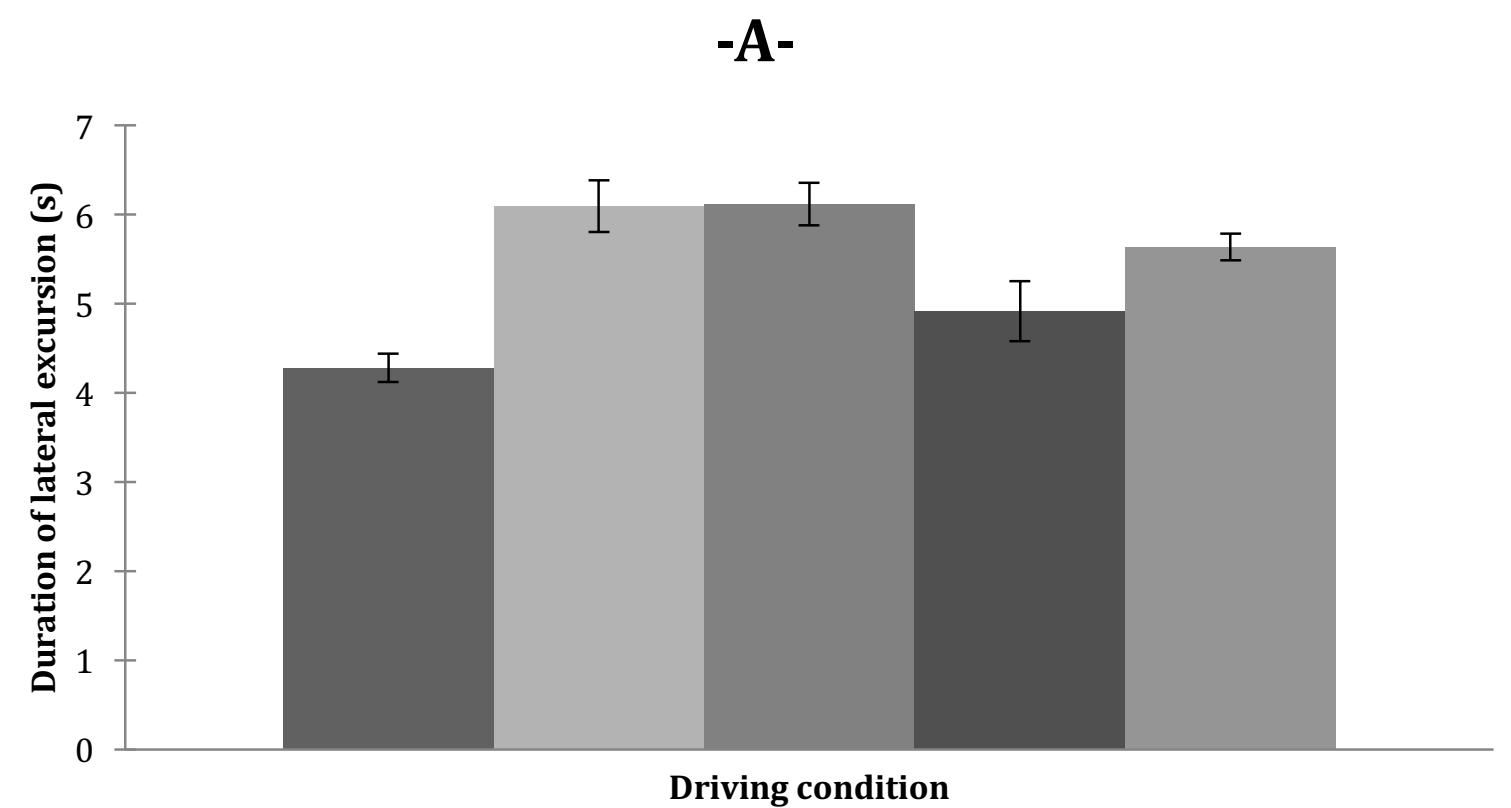

With perfectly reliable asssitance $\square$ Miss 83\% $\square$ Miss 166\% Miss $266 \%$ No assistance

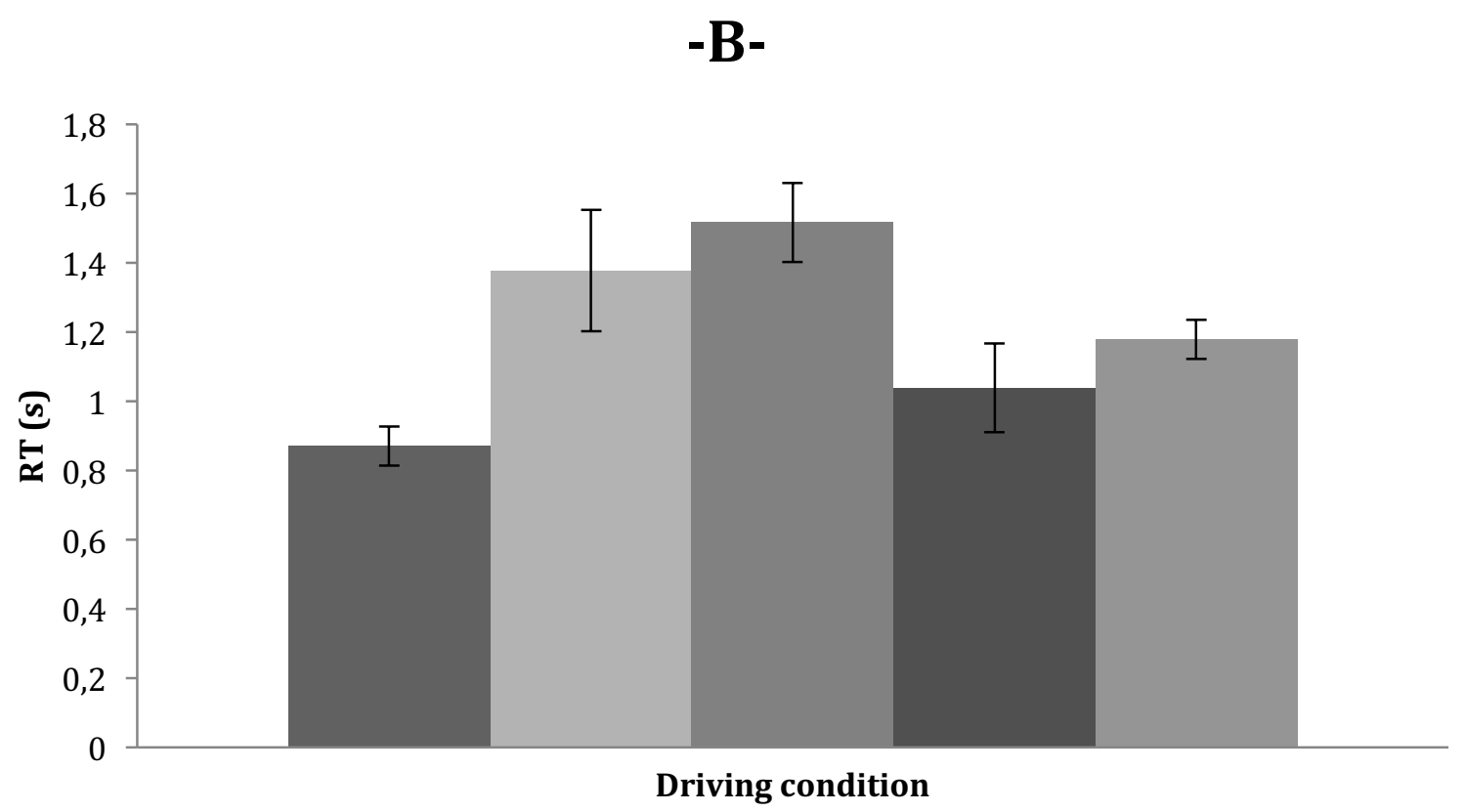

With perfectly reliable assistance $\square$ Miss $83 \% \square$ Miss $166 \%$ Miss $266 \% \square$ No assistance

Figure 2: Mean duration of lateral excursion (A) and mean steering reaction time (B) for lane departures missed compared to perfectly reliable assistance and no assistance. Error bars represent standard errors. 


\subsection{The lane departure following a missed warning}

\subsubsection{Duration of lateral excursion}

The ANOVA and associated post-hoc test pointed out a significant difference in terms of DLE between the no assistance condition and all lane departures following a missed warning (Miss 83\%, Miss 1 66\% and Miss 2 66\%) $(F(4,52)=5.32, p<.002$, Fig. 3A). DLE were significantly shorter even just after a missed warning with the assistance device than with no assistance (mean DLE after a missed warning: 4.57s, DLE without assistance: $5.64 \mathrm{~s}$ ). Furthermore, the DLE for a lane departure provoked immediately after a warning missed lane departure did not significantly differ from the DLE observed with a perfectly reliable assistance device.

\subsubsection{Steering reaction time}

The pattern of results recorded with RTs is descriptively similar to the one described for DLE (RTs in the no assistance condition longer than all lane departures following a missed warning) but failed to reach statistical significance $(F(4,52)=2.49, p<.06$, Fig. 3B). 


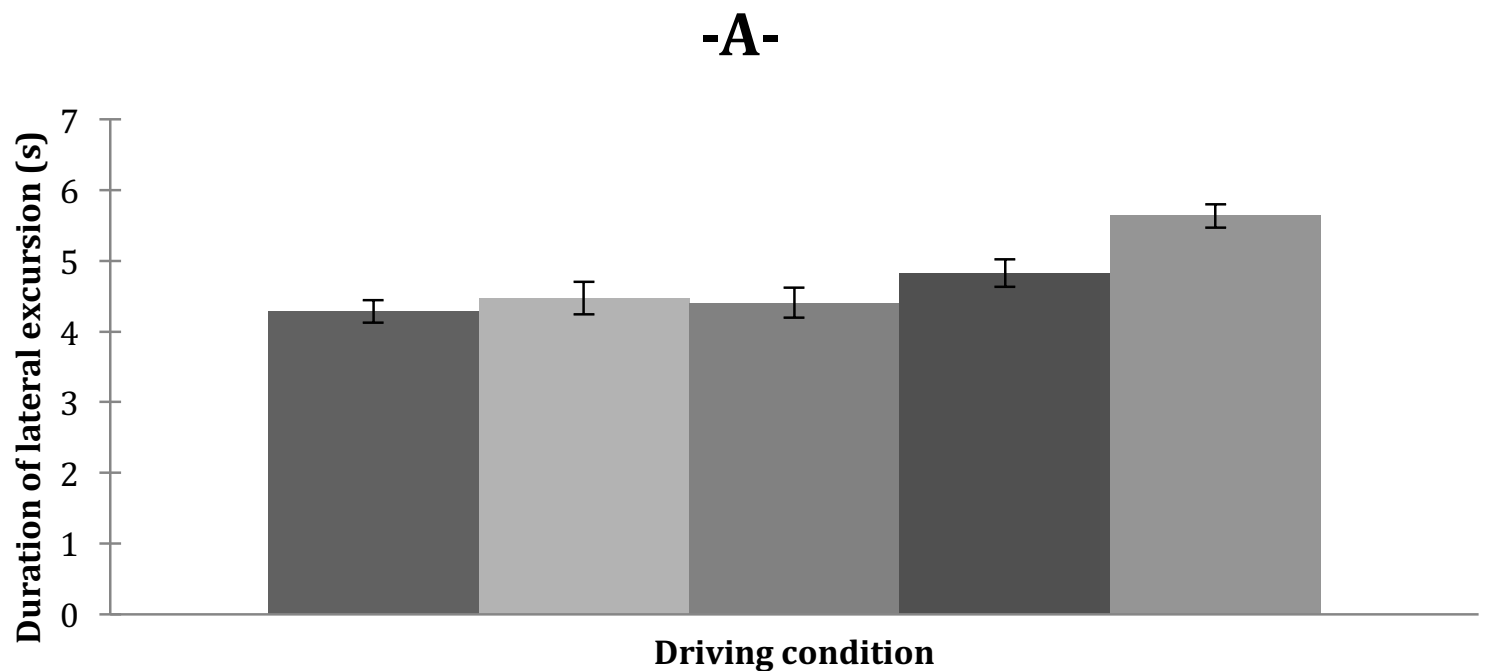

- With perfectly reliable assistance $\square$ After miss $83 \%$

- After miss $166 \%$

- After miss $266 \%$

No assistance

-B-

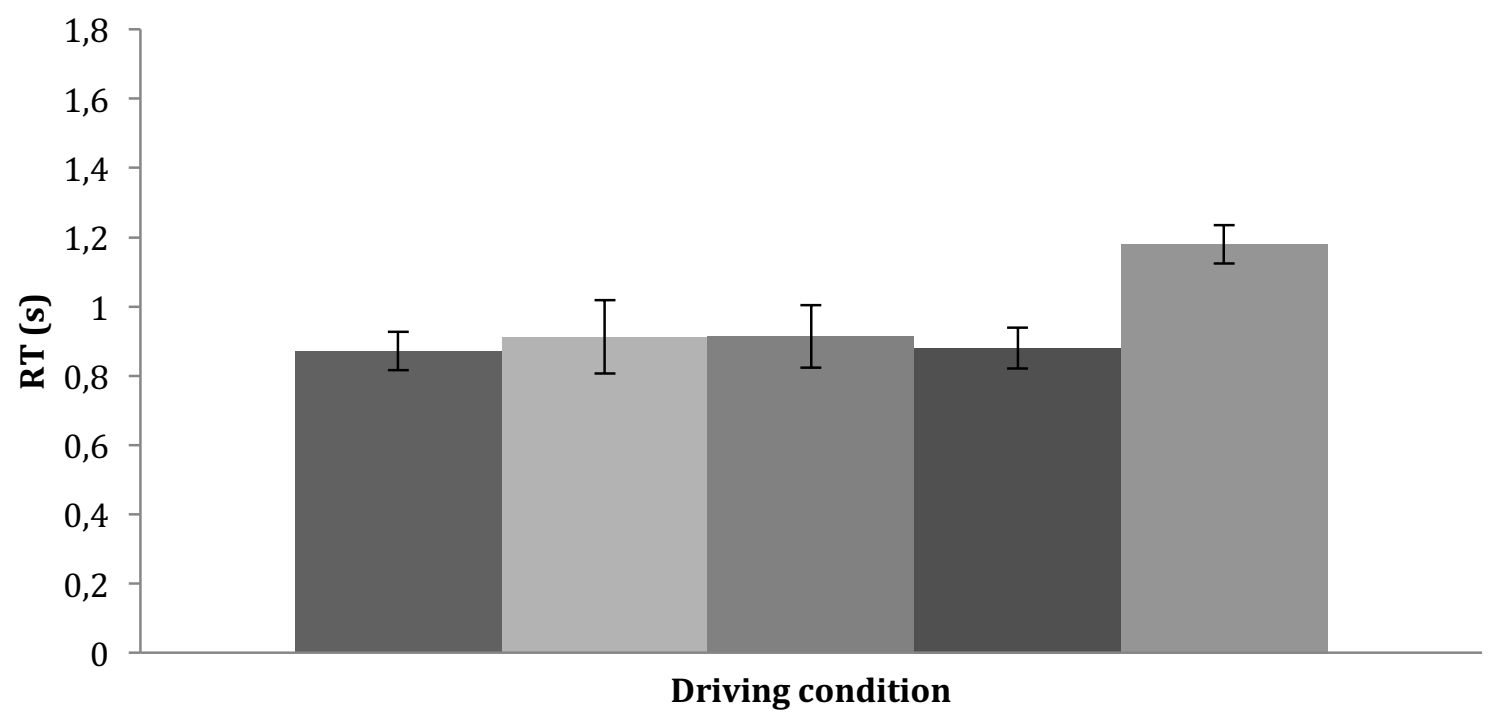

With perfectly reliable assistance $\square$ Miss $83 \% \square$ Miss $166 \% \square$ Miss $266 \% \square$ No assistance

Figure 3: Mean duration of lateral excursion (A) and mean steering reaction time (B) for the lane departure immediately following a warning missed compared to perfectly reliable assistance and no assistance. Error bars represent standard errors. 


\subsection{Global lateral position}

The assistance device reliability did not significantly influence drivers' lateral position on the driving lane $(F(2,33)=0.59, p=.56)$.

The cumulate surface around the lane center was significantly reduced in the presence of the assistance device compared to the no assistance condition (assistance: $3516 \mathrm{~m}^{2}$, no assistance: $3099 \mathrm{~m}^{2}, F(1,33)=54.42, p<.001$, Fig. 4$)$.

The onset also impacted significantly the surface around the lane center $(F(1,33)=17.15$, $p<.001)$. With FLD onset the cumulate surface around the lane center was higher $\left(3421 \mathrm{~m}^{2}\right)$ than with PLD onset $\left(3194 \mathrm{~m}^{2}\right)$.

Although the assistance triggered for PLD appeared to reduce the most the surface around the lane center, no significant interaction between the presence of the assistance and the onset was found $(F(1,33)=2.15, p=.15)$.

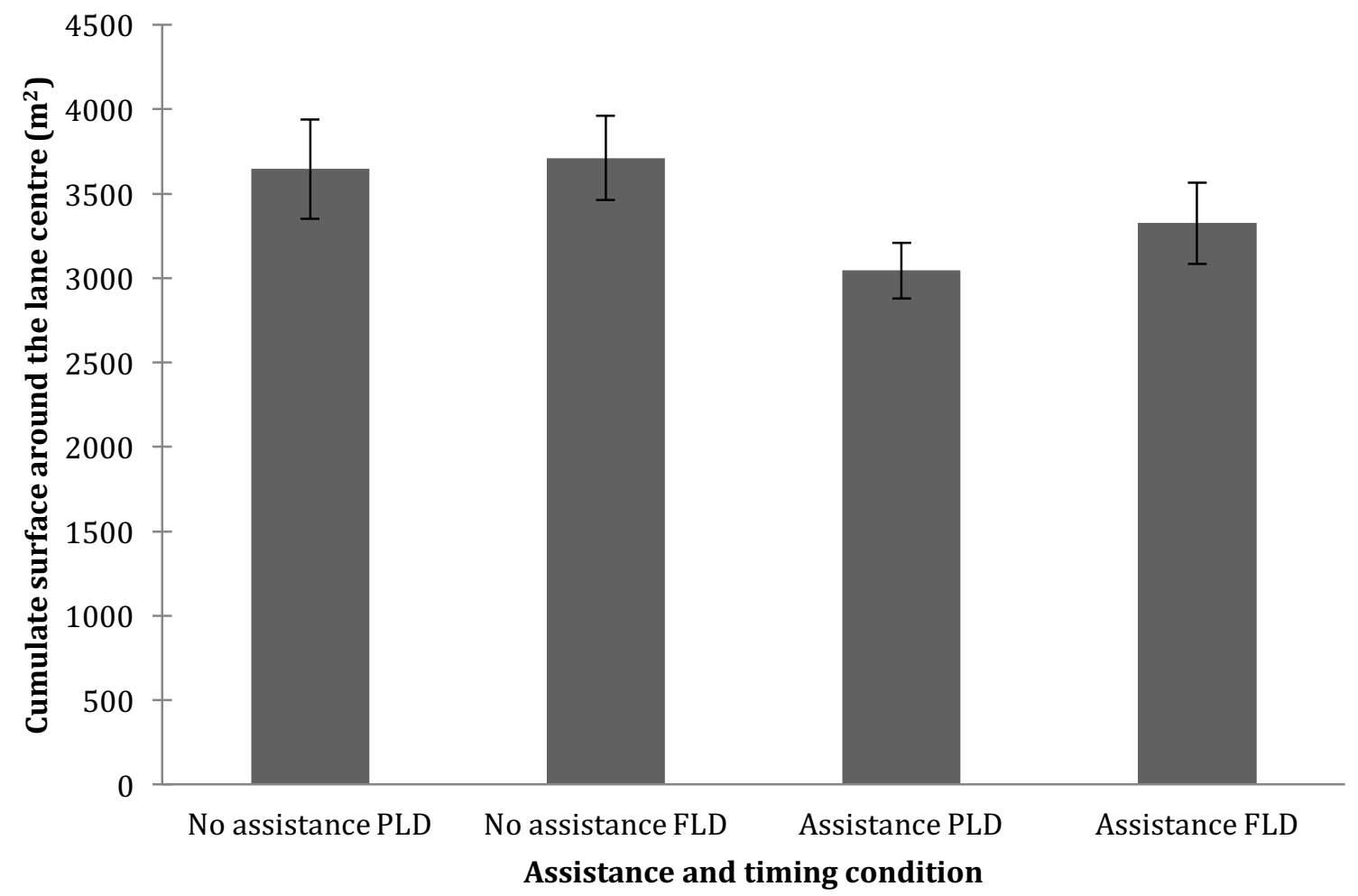

Figure 4: Cumulate surface around the lane center. Error bars represent standard errors. 


\subsection{Subjective workload and assistance acceptance}

No significant effect of the presence of assistance $(F(1,33)=0.06, p=.81)$, of the assistance reliability $(F(2,33)=1.07, p=.35)$, or onset $(F(1,33)=0.56, p=.46)$ were recorded on subjective workload.

Assistance reliability did not significantly impact any of the acceptance dimensions assessed (reliable, predictable, trustworthy, acceptable, pleasing, annoying, accurate, agreeable) and only the dimension trustworthy was significantly affected by the onset of the warning $(F(1,33)=6.42$, $p<.05)$. PLD warnings receiving a higher score (6.75 on a scale of $0-10$ ) than FLD warnings (5.94).

\section{DISCUSSION}

When looking at drivers' performances during lane departures without the warning missed situations, a global improvement was observed in the presence of the LDWS when compared with the no assistance condition. Additionally, drivers returned more quickly in their driving lane with a Partial Lane Departure than with a Full Lane Departure warning, whereas PLD or FLD did not significantly impact DLE without assistance. However, no significant reliability effect was observed. For the first lane departure missed by the assistance, drivers tended to react later and took more time to return to the driving lane when compared with properly warned lane departures. Nevertheless, recovering maneuvers were not significantly different from the no assistance condition. For the second lane departure missed, driving performances improved when compared with the first missed situation, and were in between those observed for properly warned lane departures and no assistance. Lane departures immediately following missed warnings were very similar to those observed with properly warned lane departures indicating that drivers fully benefit from the assistance in all cases. Moreover, outside of lane departure episodes, drivers swerve less in the driving lane with the LDWS than without, 
especially with the PLD onset. From a subjective point of view, LDWS did not reduce workload and PLD warnings were judged more trustworthy than the FLD ones.

The significant reduction of SRT and DLE observed without the warning missed situations confirms a general benefit of LDWS to improve drivers' performances and therefore their safety during lane departure episodes. The results extend the observations which have been made previously to unreliable LDWS when compared with perfectly reliable LDWS. Previous experiments show that perfectly reliable LDWS reduce steering reaction times (Deroo et al., 2013, 2012; Kozak et al., 2006; Navarro et al., 2007; Rossmeier et al., 2005) and decrease the number, magnitude and duration of lateral excursions (Deroo et al., 2013, 2012; Hoc et al., 2006; Navarro et al., 2010, 2007; Rimini-Döring et al., 2005). The current experiment shows that even after drivers had to deal with missed warnings, subsequent lane departures correctly warned take full advantage of the assistance in terms of reduction of both steering reaction times and duration of lateral excursion. Indeed, LDWS benefits remained stable after missed warnings, and only the recovery manoeuvers during missed warning episodes were impacted.

These findings are also consistent with those previously collected though the comparison of two levels of LDWS inaccuracy (Navarro et al., 2016). LDWS reliability ( $83 \%$ or $66 \%$ composed of false warnings alone or combined with missed warnings) did not significantly impact on the quality of the recovery maneuvers. This result is extended here even when unreliable assistance is compared with perfectly reliable assistance. This data is contrasted with some of the data collected for unreliable FCWS that led to a drop of performances (Bliss and Acton, 2003; Enriquez and MacLean, 2004). In the present experiment, the reliability of the assistance $(0 \%$, $12 \%$ or $34 \%$ of missed warnings) did not significantly affect drivers' performances and judgements of trust, other than when focusing on missed episodes. This pattern of results was also observed for FCWS in other studies with a large number of missed warnings (Maltz and Shinar, 2004) or various warning reliability levels $60 \%, 70 \%, 80 \%, 95 \%$ and $100 \%$ (Ben-yaacov et al., 2002; Bueno et al., 2013, 2012) having no significant impact on drivers behaviors. 
Moreover, even the first lane departure warned after missed warnings take full advantage of the delivered warning. In line with acceptance data indicating that drivers did not consider unreliable assistances as less trustworthy than perfectly reliable assistance. Contrary to previous data from the literature indicating a decrease in trust in automation with unreliable LDWS (Rudin-Brown and Noy, 2002), the current results indicate that trust in automation is not a key factor when it comes to LDWS. Indeed, the assistance improved recovery maneuvers as soon as a correct warning was delivered regardless of previously missed warnings.

These results are consistent with the idea that a correct warning is able to redirect drivers' attention (Ho and Spence, 2009; Ho et al., 2006, 2005; Spence and Ho, 2008) and trigger a faster reaction (Lee et al., 2002), which was observed in the present experiment through steering reaction times. LDWS impact on recovery maneuvers is extended by the assistance to diagnose the driving situation. Indeed, reductions in steering reaction times (approximately $0.3 \mathrm{~s}$ ) cannot explain alone the reduction in the duration of lateral excursion (about 1.2s) and the safety benefits associated. Results indicate that this two step warning process is not affected by the assistance reliability. The observation that missed warnings have no effect on performance as soon as the missed warning episode is over is also consistent with the idea that there is no motor memory of a steering response triggered when a warning is delivered, rather, drivers reason based on the diagnosis of the situation and adjust their behaviors accordingly (Reynaud et al., 2016).

Considering missed warning situations, if drivers' recovery maneuvers are not as good as with a valid warning, they are not significantly worse than without assistance. All together the data tends to indicate that in terms of quality of the recovery maneuvers, and associated safety benefits, an unreliable LDWS assistance would be better than no assistance. Moreover, LDWS safety benefits may also be expected outside of lane departure episodes with a general improvement of lateral control observed. In line with previous results (Rudin-Brown and Noy, 2002), drivers swerved less in the driving lane with assistance than without, regardless of the reliability level considered. This confirms the potential of LDWS (even imperfectly reliable) to 
mitigate the important transportation safety issue of lane departure crashes that represent more of $27 \%$ of crashes of light vehicles (Najm et al., 2007). With LDWS being considered as potentially relevant to prevent or mitigate between 146000 and 179000 crashes per year in the United States, including between 4842 and 7529 fatal crashes (Jermakian, 2011). Reductions in crashes have already been observed in real driving conditions, with a crash rate approximately 2 times higher for vehicles without LDWS compared to vehicles equipped with LDWS (Hickman et al., 2015).

Regarding lane departure warnings onset, drivers respond and return in their driving lane faster with PLD warnings when compared with FLD warnings, giving a clear objective advantage to the use of earlier warnings. From a subjective point of view, workload is not lowered with PLD assistance onset and only the trust dimension on an eight dimensions acceptance questionnaire is reduced (reliable, predictable, acceptable, pleasing, annoying, accurate, agreeable; Reagan \& Bliss, 2013). Contrary to what was observed with LDWS delivering false warnings alone or combined with missed warnings (Navarro et al., 2016), PLD warnings were not subjectively perceived here as more annoying and less pleasing than FLD warnings. This indicates that the nature of the assistance error is determinant on drivers' acceptance and its related use. Reinforcing the idea that false warnings are responsible for LDWS being considered as a highly annoying assistance (Braitman et al., 2010; Duan and Chen, 2011) often disengaged by drivers (Eichelberger and McCartt, 2014; Reagan and McCartt, 2016). The solution suggested here to encourage drivers to use LDWS would be to reduce as much as possible, false warnings, even it comes at the price of an increase of the risk of missed warnings. The risk of warnings given too early being considered as false warnings and therefore potentially ignored by drivers (McGehee et al., 2002; Parasuraman et al., 1997; Shinar, 1978; Tijerina et al., 1996), was not observed in the present experiment. This is probably due to the fact that even if imperfectly reliable, every warning delivered was valid. The issue of false warning annoyance being solved, earlier PLD warnings were judged as more trustworthy than 
later FLD warnings, in line with previously reported results on FCWS regarding the trust dimension (Abe and Richardson, 2006a, 2005, 2004).

An actual trend regarding transportation assistances is to automate driving as much as possible. The reason for that trend is related to our increasing technological ability to do so (Hancock, 2009). But in terms of human-machine cooperation ever increasing automation is not necessarily for the best (Parasuraman, 2000). The issue raised by always more automation is currently under debate in the human factors community; how much automation is too much? (Hancock, 2014). From a psychological perspective (i.e. drivers' perspective), increasing automation as much as possible is not the answer to all transportation safety issues. It is rather the opposite with people having a clear preference for manual completion even of mundane tasks rather than a completely automatic task completion even if it is twice as fast (Navarro and Osiurak, 2015; Osiurak et al., 2013). Additionally, the higher the degree of automation, the higher the risk of automation not only solving but also generating problems (Bainbridge, 1983). Contrary to low level assistances, high levels of driving assistance may come with incorrect use of automation (Parasuraman and Riley, 1997) and out-of-the-loop (Kaber and Endsley, 1997) or complacency phenomenon's (Parasuraman and Manzey, 2010; Parasuraman et al., 1993). As a result, warning assistances such as LDWS actually remain highly relevant to improve safety. This statement is confirmed by recent publications focusing on LDWS in various research fields (Brown et al., 2015; Dahmani et al., 2015; Huang et al., 2015; Son et al., 2015).

The collected data might also provide some guidelines to assistance designers. The study revealed that missed warnings had no significant effect on subsequent lane departure situations in terms of both driving performances and acceptance, including trust in automation. This indicates that LDWS are beneficial to drivers as soon as a valid warning is delivered. Besides when drivers faced a missed warning, this was not detrimental when compared with the no assistance condition. This indicates that when it comes to LDWS, designers should not favour the reduction of the risk of missed warning over the risk of false warnings (Parasuraman et al., 2000). Therefore, a recommendation would be to minimize the risk of false warnings. Ideally 
the warnings should be delivered only when necessary and when drivers reach a partial lane departure rather than a full lane departure.

The study may be subject to a number of potential limitations. Firstly, only few lane departure episodes were introduced and further studies are required to confirm the data in longer-term experiments. Indeed, LDWS efficiency and acceptance might change after a more prolonged period of use. Secondly, so as to ensure experimental control, lane departure episodes were induced here by means of a distraction task. This methodology may have an impact on LDWS assessment. In particular, the absence of significant changes in trust and subjective workload might be due to the fact that the distraction task was very demanding and may have masked subjective effects due to the amount of attentional resources it required. More realistic lane departures induced through drivers' drowsiness or unprovoked distraction would be valuable in order to confirm the results. Thirdly, the driver cognitive state may impact on LDWS effectiveness. In particular, the origin of the driver distraction (internal or external) could impact on the assistance effectiveness (e.g. Bueno et al., 2012). Fourthly, even if broadly used, driving simulation cannot be considered as equivalent to real driving. Drivers probably did not feel as vulnerable in the driving simulator. As a consequence, naturalist studies would be an important step so as to reinforce our conclusions.

\section{CONCLUSION}

According to the data collected, a lack of reliability with a LDWS providing valid warnings does not negatively impact drivers' performances and acceptance. Even during missed warning episodes', performances are not worse than without assistance. Interestingly, duration of lateral excursion is reduced between the first and the second warning missed. This suggests that driving performances might even be better with an assistance that is regularly missing warnings than with an assistance missing very few lane departures. In addition, driving performances for a lane departure episode following a missed warning are as good as the 
performances which were observed with a perfectly reliable assistance. The results also showed the superiority of partial lane departure warnings over full lane departure warnings, suggesting the use of partial lane departure warnings when designing LDWS. Further studies should confirm the data collected here with other lane departure inducers (e.g. with drowsy drivers) and with longer-term experiments with more lane departure episodes.

\section{ACKNOWLEDGEMENTS}

This study was supported by the French association "Fondation Sécurité Routière" (ACT project) and by the LABEX CORTEX (ANR-11-LABX-0042) of Universite de Lyon, within the program "Investissements d'Avenir" (ANR-11-IDEX-0007) operated by the French National Research Agency (ANR). The authors are very grateful to Daniel Ndiaye, Bertrand Richard and Fabien Moreau for their technical support. 
Abe, G., Itoh, M., Tanaka, K., 2002. Dynamics of drivers' trust in warning systems, in: 15th IFAC World Congress. Barcelona, Spain, July.

Abe, G., Richardson, J., 2006a. Alarm timing, trust and driver expectation for forward collision warning systems. Appl. Ergon. 37, 577-586.

Abe, G., Richardson, J., 2006b. The influence of alarm timing on driver response to collision warning systems following system failure. Behav. Inf. Technol. 25, 443-452.

Abe, G., Richardson, J., 2005. The influence of alarm timing on braking response and driver trust in low speed driving. Saf. Sci. 43, 639-654.

Abe, G., Richardson, J., 2004. The effect of alarm timing on driver behaviour: an investigation of differences in driver trust and response to alarms according to alarm timing. Transp. Res. Part F Traffic Psychol. Behav. 7, 307-322.

ADAC, 2001. Verunglückte nach Unfallart. http://www.adac.de.

Auberlet, J.-M., Pacaux, M.-P., Anceaux, F., Plainchault, P., Rosey, F., 2010. The impact of perceptual treatments on lateral control: A study using fixed-base and motion-base driving simulators. Accid. Anal. Prev. 42, 166-173.

Bainbridge, L., 1983. Ironies of automation. Automatica 19, 775-779.

Bar, F., Page, Y., 2002. Les sorties de voies involontaires. Rueil-Malmaison, F: Ceesar, LAB.

Barg-Walkow, L.H., Rogers, W.A., 2016. The Effect of Incorrect Reliability Information on Expectations, Perceptions, and Use of Automation. Hum. Factors J. Hum. Factors Ergon. Soc. 58, 242-260. doi:10.1177/0018720815610271

Ben-yaacov, A., Maltz, M., Shinar, D., 2002. Effects of an in-vehicle collision avoidance warning system on short- and long-term driving performance. Hum. Factors 44, 335342. doi: $10.1518 / 0018720024497925$

Bliss, J.P., Acton, S.A., 2003. Alarm mistrust in automobiles: how collision alarm reliability affects driving. Appl. Ergon. 34, 499-509.

Braitman, K.A., McCartt, A.T., Zuby, D.S., Singer, J., 2010. Volvo and Infiniti drivers' experiences with select crash avoidance technologies. Traffic Inj. Prev. 11, 270-8. doi:10.1080/15389581003735600

Brown, D.E., Reimer, B., Mehler, B., Dobres, J., 2015. An on-road study involving two vehicles: observed differences between an auditory and haptic lane departure warning system, in: Adjunct Proceedings of the 7th International Conference on Automotive User Interfaces and Interactive Vehicular Applications. ACM, pp. 140-145.

Bueno, M., Fabrigoule, C., Deleurence, P., Ndiaye, D., Fort, A., 2012. An electrophysiological study of the impact of a Forward Collision Warning System in a simulator driving task. Brain Res. 1470, 69-79. doi:10.1016/j.brainres.2012.06.027

Bueno, M., Fort, A., Francois, M., Ndiaye, D., Deleurence, P., Fabrigoule, C., 2013. Effectiveness of a Forward Collision Warning System in simple and in dual task from an 
electrophysiological perspective. Neurosci. Lett. 541, 219-23.

doi:10.1016/j.neulet.2013.02.030

Dahmani, H., Chadli, M., Rabhi, A., El Hajjaji, A., 2015. Vehicle dynamics and road curvature estimation for lane departure warning system using robust fuzzy observers: experimental validation. Veh. Syst. Dyn. 53, 1135-1149.

Deroo, M., Hoc, J.-M., Mars, F., 2013. Effect of strength and direction of haptic cueing on steering control during near lane departure. Transp. Res. Part F Traffic Psychol. Behav. 16, 92-103. doi:10.1016/j.trf.2012.08.015

Deroo, M., Hoc, J.-M., Mars, F., 2012. Influence of risk expectation on haptically cued corrective manoeuvres during near lane departure. Ergonomics 55, 465-475. doi:10.1080/00140139.2011.647094

Duan, L., Chen, F., 2011. The future of advanced driving assistance system development in China, in: Proceedings of 2011 IEEE International Conference on Vehicular Electronics and Safety. IEEE, pp. 238-243. doi:10.1109/ICVES.2011.5983821

Eichelberger, A.H., McCartt, A.T., 2014. Volvo drivers' experiences with advanced crash avoidance and related technologies. Traffic Inj. Prev. 15, 187-95. doi:10.1080/15389588.2013.798409

Enriquez, M., MacLean, K.E., 2004. Impact of haptic warning signal reliability in a time-andsafety-critical task, in: Haptic Interfaces for Virtual Environment and Teleoperator Systems, 2004. HAPTICS'04. Proceedings. 12th International Symposium on. IEEE, USA, pp. 407-414.

Hancock, P.A., 2014. Automation: how much is too much? Ergonomics 57, 449-454. doi:10.1080/00140139.2013.816375

Hancock, P.A., 2009. Mind, machine and morality: toward a philosophy of human-technology symbiosis. Ashgate Publishing, Ltd.

Hart, S.G., Staveland, L.E., 1988. Human Mental Workload. Adv. Psychol., Advances in Psychology 52, 139-183. doi:10.1016/S0166-4115(08)62386-9

Hatfield, J., Murphy, S., Job, R.F.S., Du, W., 2009. The effectiveness of audio-tactile lanemarking in reducing various types of crash: A review of evidence, template for evaluation, and preliminary findings from Australia. Accid. Anal. Prev. 41, 365-379.

Hickman, J.S., Guo, F., Camden, M.C., Hanowski, R.J., Medina, A., Mabry, J.E., 2015. Efficacy of roll stability control and lane departure warning systems using carriercollected data. J. Safety Res. 52, 59-63. doi:10.1016/j.jsr.2014.12.004

Ho, C., Spence, C., 2009. Using Peripersonal Warning Signals to Orient a Driver's Gaze. Hum. Factors J. Hum. Factors Ergon. Soc. 51, 539-556. doi:10.1177/0018720809341735

Ho, C., Tan, H.Z., Spence, C., 2006. The differential effect of vibrotactile and auditory cues on visual spatial attention. Ergonomics 49, 724-738.

Ho, C., Tan, H.Z., Spence, C., 2005. Using spatial vibrotactile cues to direct visual attention in driving scenes. Transp. Res. Part F Traffic Psychol. Behav. 8, 397-412.

Hoc, J.-M., Mars, F., Milleville-Pennel, I., Jolly, É., Netto, M., Blosseville, J.-M., 2006. Human-machine cooperation in car driving for lateral safety: delegation and mutual control. Trav. Hum. 69, 153-182. 
Hoc, J.-M., Young, M.S., Blosseville, J.-M., 2009. Cooperation between drivers and automation: implications for safety. Theor. Issues Ergon. Sci. 10, 135-160.

Huang, Z., Wu, Y., Liu, J., 2015. Research on effects of pattern, amplitude and frequency of pulse steering torque warnings for lane departure. Transp. Res. Part F Traffic Psychol. Behav. 31, 67-76. doi:10.1016/j.trf.2015.03.008

Jermakian, J.S., 2011. Crash avoidance potential of four passenger vehicle technologies. Accid. Anal. Prev. 43, 732-740. doi:10.1016/j.aap.2010.10.020

Kaber, D.B., Endsley, M.R., 1997. Out-of-the-loop performance problems and the use of intermediate levels of automation for improved control system functioning and safety. Process Saf. Prog. 16, 126-131.

Kovordányi, R., Ohlsson, K., Alm, T., 2005. Dynamically deployed support as a potential solution to negative behavioral adaptation, in: Proceedings of the Intelligent Vehicles Symposium Sponsored by the IEEE Intelligent Transportation Systems Society. IEEE, Portugal, pp. 613-618.

Kozak, K., Pohl, J., Birk, W., Greenberg, J., Artz, B., Blommer, M., Cathey, L., Curry, R., 2006. Evaluation of lane departure warnings for drowsy drivers, in: Proceedings of the Human Factors and Ergonomics Society Annual Meeting. Sage Publications, pp. 24002404.

Land, M.F., Horwood, J., 1995. Which parts of the road guide steering? Nature 377, 339-340.

Lee, J., Moray, N., 1992. Trust, control strategies and allocation of function in humanmachine systems. Ergonomics 35, 1243-1270.

Lee, J.D., McGehee, D., Brown, T.L., Reyes, M.L., 2002. Collision warning timing, driver distraction, and driver response to imminent rear-end collisions in a high-fidelity driving simulator. Hum. Factors 44, 314-334.

Lété, B., Sprenger-Charolles, L., Colé, P., 2004. MANULEX: A grade-level lexical database from French elementary school readers. Behav. Res. Methods 36, 156-166.

Maltz, M., Shinar, D., 2007. Imperfect in-vehicle collision avoidance warning systems can aid distracted drivers. Transp. Res. part F traffic Psychol. Behav. 10, 345-357.

Maltz, M., Shinar, D., 2004. Imperfect in-vehicle collision avoidance warning systems can aid distracted drivers. Hum. Factors 46, 357-366.

McGehee, D. V, Brown, T.L., Lee, J.D., Wilson, T.B., 2002. Effect of warning timing on collision avoidance behavior in a stationary lead vehicle scenario. Transp. Res. Rec. J. Transp. Res. Board 1803, 1-6.

Meuleners, L.B., Hendrie, D., Lee, A.H., 2011. Effectiveness of sealed shoulders and audible edge lines in Western Australia. Traffic Inj. Prev. 12, 201-205.

Morena, D.A., 2003. The nature and severity of drift-off road crashes on Michigan freeways, and the effectiveness of various shoulder rumble strip designs, in: Proceedings of TRB 2003 Annual Meeting. pp. 1-23.

Muir, B.M., 1994. Trust in automation: Part I. Theoretical issues in the study of trust and human intervention in automated systems. Ergonomics 37, 1905-1922.

Najm, W.G., Smith, J.D., Yanagisawa, M., 2007. Pre-crash scenario typology for crash avoidance research, (Tech. Rep. DOT-HS-810 767). National Highway Transportation 
Safety Administration Research, United States.

National Highway Traffic Safety Administration, 2006. Traffic safety facts 2005. U.S. Department of Transportation, Washington.

Navarro, J., Mars, F., Forzy, J.-F., El-Jaafari, M., Hoc, J.-M., 2010. Objective and subjective evaluation of motor priming and warning systems applied to lateral control assistance. Accid. Anal. Prev. 42, 904-12. doi:10.1016/j.aap.2009.07.008

Navarro, J., Mars, F., Hoc, J.-M., 2007. Lateral control assistance for car drivers: a comparison of motor priming and warning systems. Hum. Factors 49, 950-60.

Navarro, J., Mars, F., Young, M.S., 2011. Lateral control assistance in car driving: classification, review and future prospects. IET Intell. Transp. Syst. 5, 207-220. doi:10.1049/iet-its.2010.0087

Navarro, J., Osiurak, F., 2015. When do use automatic tools rather than doing a task manually? Influence of automatic tool speed. Am. J. Psychol. 128, 77-88.

Navarro, J., Yousfi, E., Deniel, J., Jallais, C., Bueno, M., Fort, A., 2016. The impact of false warnings on partial and full lane departure warnings effectiveness and acceptance in car driving. Ergonomics 1-30. doi:10.1080/00140139.2016.1158323

Osiurak, F., Wagner, C., Djerbi, S., Navarro, J., 2013. To do it or to let an automatic tool do it? The priority of control over effort. Exp. Psychol. 60, 453.

Parasuraman, R., 2000. Designing automation for human use: empirical studies and quantitative models. Ergonomics 43, 931-951.

Parasuraman, R., Hancock, P.A., Olofinboba, O., 1997. Alarm effectiveness in driver-centred collision-warning systems. Ergonomics 40, 390-399.

Parasuraman, R., Manzey, D.H., 2010. Complacency and bias in human use of automation: an attentional integration. Hum. Factors 52, 381-410. doi:10.1177/0018720810376055

Parasuraman, R., Masalonis, A.J., Hancock, P.A., 2000. Fuzzy signal detection theory: basic postulates and formulas for analyzing human and machine performance. Hum. Factors 42, 636-659. doi:10.1518/001872000779697980

Parasuraman, R., Molloy, R., Singh, I.L., 1993. Performance consequences of automationinduced'complacency'. Int. J. Aviat. Psychol. 3, 1-23.

Parasuraman, R., Riley, V., 1997. Humans and Automation : Use, Misuse, Disuse , Abuse. Hum. Factors J. Hum. Factors Ergon. Soc. 39, 230-253. doi:10.1518/001872097778543886

Pohl, J., Birk, W., Westervall, L., 2007. A driver-distraction-based lane-keeping assistance system. Proc. Inst. Mech. Eng. Part I J. Syst. Control Eng. 221, 541-552.

Reagan, I.J., Bliss, J.P., 2013. Perceived mental workload, trust, and acceptance resulting from exposure to advisory and incentive based intelligent speed adaptation systems. Transp. Res. Part F Traffic Psychol. Behav. 21, 14-29. doi:10.1016/j.trf.2013.07.005

Reagan, I.J., McCartt, A.T., 2016. Observed Activation Status of Lane Departure Warning and Forward Collision Warning of Honda Vehicles at Dealership Service Centers. Traffic Inj. Prev. 9588, 00-00. doi:10.1080/15389588.2016.1149698

Reynaud, E., Lesourd, M., Navarro, J., Osiurak, F., 2016. On the NeuroCognitive Origins of Human Tool Use A Critical Review of NeuroImaging Data. Neurosci. Biobehav. Rev. 
doi:10.1016/j.neubiorev.2016.03.009

Rimini-Döring, M., Altmueller, T., Ladstaetter, U., Rossmeier, M., 2005. Effects of lane departure warning on drowsy drivers' performance and state in a simulator, in:

Proceedings of the Third International Driving Symposium on Human Factors in Driver Assessment, Training, and Vehicle Design. pp. 88-95.

Robinson, E., Lerner, N., Singer, J., Jenness, J., Traube, E., 2011. Comprehension of Advanced Collision Warning System Displays, in: 18th ITS World Congress.

Rossmeier, M., Grabsch, H.-P., Rimini-Döring, M., 2005. Blind flight: Do auditory lane departure warnings attract attention or actually guide action, in: Proceedings of the 11th Meeting of the International Conference on Auditory Display.

Rudin-Brown, C.M., Noy, I.Y., 2002. Investigation of behavioral adaptation to lane departure warnings. Transp. Res. Rec. J. Transp. Res. Board 1803, 30-37.

Shinar, D., 1978. Psychology on the Road. The Human Factor in Traffic Safety, Wiley. ed. New York.

Son, J., Yoo, H., Kim, S., Sohn, K., 2015. Real-time illumination invariant lane detection for lane departure warning system. Expert Syst. Appl. 42, 1816-1824.

Spence, C., Ho, C., 2008. Multisensory warning signals for event perception and safe driving. Theor. Issues Ergon. Sci. 9, 523-554. doi:10.1080/14639220701816765

Stanton, N.A., Young, M.S., Walker, G.H., Turner, H., Randle, S., 2001. Automating the driver's control tasks. Int. J. Cogn. Ergon. 5, 221-236. doi:10.1207/S15327566IJCE0503_5

Tijerina, L., Jackson, J.L., Pomerleau, D.A., Romano, R.A., Petersen, A.D., 1996. Driving simulator tests of lane departure collision avoidance systems, in: Intelligent Transportation: Realizing the Benefits. Proceedings of the 1996 Annual Meeting of ITS America.

Wickens, C.D., Dixon, S.R., 2007. The benefits of imperfect diagnostic automation: A synthesis of the literature. Theor. Issues Ergon. Sci. 8, 201-212.

Young, M.S., Stanton, N.A., Harris, D., 2007. Driving automation: learning from aviation about design philosophies. Int. J. Veh. Des. 45, 323-338. 